\title{
Can the mini-sling become the golden standard for treating stress urinary incontinence?
}

\author{
Cheng-Yu Long ${ }^{1} \cdot$ Gin-Den Chen ${ }^{2} \cdot$ Rebecca G. Rogers ${ }^{3}$
}

Received: 23 November 2019 / Accepted: 25 January 2020 / Published online: 26 November 2020

(C) The International Urogynecological Association 2020

The evolution of anti-incontinence surgeries is dramatic, and innovative paradigms change frequently. In the late 1990s, retropubic midurethral slings overtook Burch's colposuspension to become the gold standard for surgical treatment of female urinary incontinence (SUI). In the past 2 decades, the second generation of the mid-urethral sling, transobturator tape, has proved to be as effective as retropubic midurethral slings with fewer intraoperative complications. Recently, the third generation of the mid-urethral sling, the mini-sling (using less mesh), has attracted attention because this procedure avoids passing trocars through the space of Retzius or through the obturator foramen. Theoretically, these procedures avoid bladder perforation and major vessel injury, which may occur during retropubic procedures, and postoperative thigh and groin pain, which may occur after transobturator procedures.

Two randomized clinical trials comparing the mini-sling to TVT-O published in the IUJ reveal similar efficacy, safety and mesh erosion rates between the two procedures. In addition, the trials found that women who underwent a mini-sling procedure reported lower intensity and a shorter duration of postoperative pain after 2 and 3 years of follow-up [1,2]. Length of vaginal tape $(12 \mathrm{~cm}$ vs. $19 \mathrm{~cm}$ ) seems to be the main factor that influences the amount of postoperative pain. This may be due to less neuro-muscular injury by reducing the depth of

Gin-Den Chen

gdchentw@hotmail.com

1 Department of Obstetrics and Gynecology, Kaohsiung Municipal Siaogung Hospital, Kaohsiung, Taiwan

2 Department of Obstetrics and Gynecology, Chung Shan Medical University Hospital, 110, Jianguo N Rd, Section 1, Taichung 402, Taiwan

3 Department of Women's Health, Dell Medical School, The University of Texas at Austin, Austin, TX, USA lateral dissection, avoiding perforation of the obturator membrane with scissors or a guide and the presence of the tape beneath the groin. One study used the Pelvic Organ Prolapse/ Urinary Incontinence Sexual Questionnaire to evaluate sexual function after surgery and found that sexually active women had improved sexual function and no significant change in pain during intercourse following these procedures.

Mesh exposure following its use is important. In these two trials, rates of mesh exosure for the mini-sling were $2 \%$ and $4.9 \%$ in 3 years of follow-up, respectively [2, 3]. Another 24month follow-up study showed $3.37 \%$ of mesh exposure was $\leq 1 \mathrm{~cm}$, and $2.24 \%$ was $>1 \mathrm{~cm}$ according to the IUGA/ICS Classification of Prosthesis-related Complications [1]. The mesh exposure rates of this product are similar to those of the classical mid-urethral sling in mid-term follow-up. However, the questions that remain are whether these mesh exposure rates are currently acceptable and whether the mesh exposure rates would increase in longer follow-up periods.

Data on the mini-sling continue to evolve. Another article in the IUJ this year showed that the mini-sling is an effective treatment for urodynamic stress incontinence after a 3-year follow-up, and the incidence of de novo overactive bladder was $4.9 \%$ and $15 \%$ at the 1 - and 3-year follow-up, respectively. There were no serious late complications, and no tape revision or resection was required over 3 years [3].

Based on these and other findings, the mini-sling may survive a "high vigilance restriction period" that some national health care systems have endorsed for mesh products including the ban of synthetic mesh for transvaginal pelvic floor reconstruction in the US and the ban of the midurethral slings for treating primary stress urinary incontinence in the UK [4]. Large-scale studies with longterm follow-up periods are needed to clarify the role of the mini-sling in the treatment of SUI. Whether the mini-sling will become the new gold standard for anti-incontinence surgery remains to be seen. 


\section{Compliance with ethical standards}

\section{Conflict of interest None.}

\section{References}

1. Dogan O, Kaya AE, Pulatoglu C, Basbug A, Yassa M. A randomized comparison of a single-incision needless (Contasure-needleless ${ }^{\circledR}$ ) mini-sling versus an inside-out transobturator (Contasure-KIM®) mid-urethral sling in women with stress urinary incontinence: 24month follow-up results. Int Urogynecol J. 2018;29:1387-95.
2. Zullo MA, Schiavi MC, Luffarelli P, Prata G, Di Pinto A. Oliva C. TVT-O vs TVT-Abbrevo for stress urinary incontinence treatment in women: a randomized trial Int Urogynecol J. 2019. https://doi.org/ 10.1007/s00192-019-04077-7.

3. Braga A, Caccia G, Ruggeri G, Regusci L, Papadia A, Serati M. 3year follow-up of tension-free vaginal tape-ABBREVO procedure for the treatment of pure urodynamic stress urinary incontinence: efficacy and adverse effects. Int Urogynecol J. 2018;29:1387-95.

4. Swift S. What if you could not do a mid-urethral sling? 2019;30: 1387-1388.

Publisher's note Springer Nature remains neutral with regard to jurisdictional claims in published maps and institutional affiliations. 